\title{
Epidemiology and survival analyses of 333 adult glioma patients from Eastern Algeria (2008-2016)
}

\author{
Sabrina Touati ${ }^{1}$, Rachid Djekkoun ${ }^{2,3}$, Mohamed El-Hadef El-Okki ${ }^{4}$, Dalila Satta ${ }^{1}$
}

1. Laboratory of molecular and cellular biology, Mentouri Brothers University, Constantine, Algeria.

2. Radiation-Oncology Department, University Hospital Benbadis, Constantine, Algeria.

3. Occupational hazards and health laboratory, Salah Boubnider University, Constantine, Algeria.

4. Biology and Environment Laboratory, Mentouri Brothers University, Constantine, Algeria.

\section{Authors adresses:}

Rachid Djekkoun: rdjekkoun@gmail.com; Mohamed El-Hadef El-Okki:

elhadef-elokki.mohamed@umc.edu.dz/http://orcid.org/0000-0002-6854-1925; Dalila Satta: dsatta741@gmail.com

\begin{abstract}
:
Background: Gliomas are a relatively rare group of tumors with a poor prognosis. We aimed to describe and analyze the clinical characteristics and survival of patients with glioma tumors of Eastern Algeria.

Methods: A retrospective study was conducted at the University Hospital of Constantine. Medical records of patients enrolled between January 2008 and October 2016 were consulted. Demographic characteristics, clinical data, treatment strategy and dates of last follow-up or death were collected. Chi-square test was used for checking associations, KaplanMeier methodology for estimating the survival, and the cox model for identifying prognosis factors.

Results: A total of 333 patients composed our cohort. The mean age was 48.07 years, and men were 1.87 times more frequent than women. High grade tumors were mainly observed among adults and old adults and in supra-tentorial locations. More than half of the patients had a large resection and a curative protocol of oncological treatment $(50.7 \%$ and $57 \%$, respectively). The mean overall survival was 45.4 months, the median was 21.7 months, and survival rates at 1-, 2-, and 5-years were: $62.8 \%, 48.5 \%$ and $32.9 \%$ respectively. Age, histology, grade of malignancy and oncological treatment were the major prognosis factors.
\end{abstract}

Conclusion: Our sample was relatively young with a higher survival compared to others.

Keywords: Glioma; epidemiology; Algeria.

DOI: https://dx.doi.org/10.4314/ahs.v20i3.29

Cite as: Touati S, Djekkoun R, El-Okeki ME-H, Satta D. Epidemiology and survival analyses of 333 adult glioma patients from Eastern Algeria (2008-2016). Afri Health Sci. 2020;20(3): 1250-1258. bttps:// dx.doi.org/10.4314/ahs.v20i3.29

\section{Introduction}

Gliomas are a heterogeneous group of neoplasms that starts at the glial cells in the Central Nervous System (CNS). These tumors are relatively rare but highly aggressive $^{1,2}$. As reported by the latest worldwide statistics of cancer (GLOBOCAN 2018), brain and CNS tumors, including all types, represent $1.6 \%$ of new cancers and $2.5 \%$ of cancer deaths ${ }^{3}$. Gliomas constitute about $26.5 \%$ of primary brain and CNS tumors, and $80.7 \%$ of malignant tumors ${ }^{4}$.

\section{Corresponding author:}

Sabrina Touati,

Laboratory of molecular and cellular biology,

Faculty of Natural and Life Sciences,

Department of Animal Biology,

Mentouri Brothers University, Constantine, Algeria

E-mail : Sabrina_touati@umc.edu.dz
According to the World Health Organization (WHO), gliomas are classified into different histological subtypes depending on the glial cell of origin (eg : astrocyte, oligodendrocyte), and into 4 grades of malignancy (from I to IV) ${ }^{5}$. In fact, the prognosis of gliomas represents a substantial challenge. Despite the advances in clinical care, the outcome of patients remains globally poor; the 5 -year overall survival (OS) for glial tumors is less than $20 \%$, and for glioblastoma multiform (GBM), the most frequent subtype, it is about $5 \%{ }^{4,6}$. Survival generally decreases with age at the time of diagnosis. Children and young adults commonly have a higher survival rate for most histological types ${ }^{4}$.

Epidemiological studies have largely contributed to the understanding of these pathologies, particularly in developed countries. The latest report of the (WHO) estimated that the highest rates of incidence and mortality of cancers are recorded in the most developed regions

2020 Touati S et al. Licensee African Health Sciences. This is an Open Access article distributed under the terms of the Creative commons Attribution License (https://creativecommons.org/licenses/BY/4.0), which permits unrestricted use, distribution, and reproduction in any medium, provided the original work is properly cited. 
and are lower in developing countries. The reason of this disparity may be due to the fact that the African and Asian populations have a modest coverage by cancer registries (11\% and $8 \%$ respectively), or the availability of diagnostic facilities, or the inaccuracy of attribution of the cause of cancer death ${ }^{7}$. Data on populations of developing countries remains generally limited, as it is the case in Algeria, where little is known about the epidemiology of gliomas. The observational study of Hamdi Cherif et al, based on the cancer registry of Setif (East of Algeria), noted that the overall cancer incidence rates are increasing in both sexes, and that the age-standardized rates per 100,000 (to the world standard population) are 106.4 in men and 110.3 in women, however, no statistics about brain and CNS tumors are reported ${ }^{8}$. Furthermore, the program "Cancer Plan 2015-2019" accomplished in Algeria, which aimed to reduce cancer mortality with an improvement of the preventive approach against risk factors and quality of life during and after treatment, focused on the common types of cancer.

The present study aimed to describe and analyze the clinical characteristics and survival rate of adult glioma patients in Eastern Algeria.

\section{Materials and methods}

The present single center retrospective study was conducted in the Radiation-Oncology Department of Anti-Cancer Center at the University Hospital Benbadis, Constantine, where the majority of cancer patients from Eastern Algeria are received.

\section{Data collection}

The data were obtained from medical records of adult patients with glial tumors who were admitted between January 2008 and October 2016. The histological classifications were based on the 2007 WHO classification standard of brain tumors ${ }^{5}$. The study included the following histological subtypes: Astrocytomas, Oligodendrogliomas, Glioblastomas (GBM), Mixed tumors, and Gliomas Not Otherwise Specified (NOS).

The study was carried out on a total of 425 records. The demographic characteristics (age and sex); clinical data (tumor location, histological subtype and grade of malignancy); treatment strategies (type of surgery and type of oncological treatment) and detailed information about the survival of the patients, including time of surgery, histopathological diagnosis and the time of the last follow-up) were collected and registered in an Excel File. Records with missing data were excluded from the study, and a total of 333 patients composed the cohort. The study was concluded in March 2017.

\section{Statistical analyses}

Statistical analyses were performed using $\mathrm{R}$ software (R-3.4.3). Chi-square ( $\chi 2)$ test was used to assess the association between the independent variables: age group, sex, tumor location, histological subtype and grade of malignancy (excluding the test between the two last ones). Kaplan-Meier methodology was used to estimate survival rates, mean and median survival time and 95\% confidence interval. The overall survival (OS) was defined as the time period from the day after surgery until death or until the last follow-up. Univariable and multivariable analyses, using the Cox-proportional hazard model, were performed to identify prognosis factors. A value of $p$ less than 0.05 was considered statistically significant. Proportional hazards assumption was checked using the Schoenfeld residuals. Since the assumption was violated, the model was refitted by an Extended Cox-PH.

\section{Results}

Adults glioma patients registered in this study represent $2.65 \%(\mathrm{n}=333)$ of the 12537 cancer patients who were admitted to the Radiation-Oncology Department of the Anti-Cancer Center in the same period. The distribution of patients according to the sex, age group, location, histology and grade is shown in Table 1.

\section{Demographic features}

Among a cohort of 333 glioma patients, $65 \%$ were male and $35 \%$ were female with a sex ratio male / female of 1.87. The mean age at diagnosis was $48.07 \pm 13.5$ years ranging from 20 to 78 (median $=49$ ). For the statistical analysis purpose, the patients were categorized into three age groups: Young adults (aged between 20 and 39 years old), Adults (40 to 59 years), and Old adults (60 years and older). The adult group had the highest rate with $51.3 \%$ of the cases.

\section{Location}

The main location of tumors in our population was brain lobes in $88.5 \%(\mathrm{n}=295)$ of the cases. The left hemisphere was affected in $48.9 \%(n=163)$ of the patients. The other locations were less frequent, as the cerebellum which was affected in 6 cases, the brainstem was the location in 5 patients and the ventricles in 4 patients. For statistical analysis, tumors were assembled as shown in Table 1: Supra-tentorial, Infra-tentorial and Multifocal. 


\section{Statistical analysis}

The $\chi^{2}$ test has revealed that the grade of malignancy increases with age $(p<0.001)$, that high grade tumors (grade III and IV) were mainly supra tentorial located $(p<0.001)$ and that infra tentorial locations were the most observed among the youngest patients $(p<0.01)$.

\section{Survival analysis}

The mean follow-up time was 17.4 months, the median was 9 months, and $42.9 \%(n=143)$ of the patients had died by the time statistical analyses started. The mean OS was 45.4 months, the median 21.7 months and 95\% Confidence Interval (CI) [14.13 - 37.07]. Survival rates at $1-, 2$, and 5 -years for the entire population were: $62.8 \%, 48.5 \%$ and $32.9 \%$ respectively.

Table 1 Demographic, clinical characteristics and survival of glioma patients

\begin{tabular}{|c|c|c|c|c|c|c|}
\hline \multirow[b]{2}{*}{ Variables } & \multirow[b]{2}{*}{ Groups } & \multirow[b]{2}{*}{$\mathrm{N}(\%)$} & \multicolumn{4}{|c|}{ Survival } \\
\hline & & & $\begin{array}{l}\text { Mean OS } \\
\text { (Months) } \\
\end{array}$ & lyear & 2years & 5 years \\
\hline \multirow[t]{2}{*}{ Sex } & Men & $217(65)$ & 42.6 & $61.2 \%$ & $46.8 \%$ & $31.1 \%$ \\
\hline & Women & $116(35)$ & 48.8 & $65.8 \%$ & $52 \%$ & $37 \%$ \\
\hline \multirow{3}{*}{$\begin{array}{l}\text { Age group } \\
\text { (years) }\end{array}$} & Young adults (20-39) & $91(27.3)$ & 69 & $87.1 \%$ & $79.8 \%$ & $56.3 \%$ \\
\hline & Adults (40-59) & $171(51.3)$ & 38.1 & $57.8 \%$ & $43.3 \%$ & $28.6 \%$ \\
\hline & Old adults $(\geq 60)$ & $71(21.3)$ & 14.3 & $36.3 \%$ & $0 \%$ & $0 \%$ \\
\hline \multirow[t]{3}{*}{ Location } & Supra-tentorial & $306(91.9)$ & 40.5 & $61.4 \%$ & $46.5 \%$ & $27.9 \%$ \\
\hline & Infra-tentorial & $18(5.4)$ & 85 & $87.1 \%$ & $80.4 \%$ & $80.4 \%$ \\
\hline & Multifocal & $9(2.7)$ & 30.4 & $52.5 \%$ & $35 \%$ & $17.5 \%$ \\
\hline \multirow[t]{5}{*}{ Histology } & Glioblastoma & $183(55)$ & 13.4 & $42.7 \%$ & $14.2 \%$ & $00 \%$ \\
\hline & Astrocytoma & $83(25)$ & 56.4 & $76.1 \%$ & $70.3 \%$ & $56.9 \%$ \\
\hline & Oligodendroglioma & $31(9)$ & 64 & $100 \%$ & $94.7 \%$ & $46.6 \%$ \\
\hline & Mixed tumors & $29(9)$ & 64 & $80.5 \%$ & $75.7 \%$ & $68.8 \%$ \\
\hline & Glioma NOS & $7(2)$ & 47.8 & $66.7 \%$ & $66.7 \%$ & $44.4 \%$ \\
\hline \multirow[t]{4}{*}{ Grade } & Grade I & $13(4)$ & 81.2 & $100 \%$ & $81.8 \%$ & $81.8 \%$ \\
\hline & Grade II & $65(19.5)$ & 75.6 & $90.9 \%$ & $90.9 \%$ & $61.2 \%$ \\
\hline & Grade III & $72(21.6)$ & 57.9 & $71.3 \%$ & $65 \%$ & $47.1 \%$ \\
\hline & Grade IV & $183(55)$ & 13.4 & $42.7 \%$ & $14.2 \%$ & $00 \%$ \\
\hline
\end{tabular}

Abbreviations: $\mathrm{OS}=$ overall survival; NOS $=$ not otherwise specified

The global median survival in adults was 16 months. It was 21 months in patients with supratentorial tumors, 62 months in astrocytoma and 54 months in oligodendroglioma. In the high-grade gliomas, the lowest survival was registered among GBM (GIV) patients with 11 months, while Grade III tumors had a median survival of 54 months.

\section{Treatment}

As shown in Table 2, treatment scheme was divided into: "Surgery: large resection, partial resection and biopsy" and "Oncological treatment: curative, palliative and not treated". The class "not treated" included patients who died before or during the Radiotherapy. 
Table 2 Distribution of Glioma patients according to treatment received

\begin{tabular}{lccc|c}
\hline & $\begin{array}{c}\text { Large } \\
\text { resection }\end{array}$ & $\begin{array}{c}\text { Partial } \\
\text { resection }\end{array}$ & Biopsy & Total \\
\hline Curative & 103 & 63 & 24 & $190(57 \%)$ \\
Palliative & 31 & 25 & 15 & $71(21.3 \%)$ \\
$\begin{array}{l}\text { Not } \\
\text { treated }\end{array}$ & 35 & 19 & 18 & $72(21.6 \%)$ \\
\hline Total & $169(50.7 \%)$ & $107(32.1 \%)$ & $57(17.1 \%)$ & 333
\end{tabular}

According to the type of surgery, the mean OS for patients who underwent a biopsy was 39.6 months; it was of 40.5 months for those who had a large resection and, surprisingly, 53.2 months for those who had a partial resection. On the other hand, patients who received curative protocol of oncological treatment lived on average 57.6 months; those who received palliative protocol had a mean OS of 20.2 months; and it was 13.9 months for those classified as not treated.

Regarding oncological treatment, 190 patients of the curative protocol received 3D conformal radiotherapy whose total dose varied between 40 and 60 Gray (Gy). One hundred and twenty-one (121) patients received exclusive radiation therapy, and sixty-nine (69) received chemotherapy combined with radiation therapy. Sixty-one (61) patients received Temozolomide as chemotherapy and the other 8 received 3 courses of cisplatin/ etoposide.
The 71 patients concerned by palliative treatment benefited from 3D conformal radiotherapy hypo-fractionated according to several schemes; a single series of $18 \mathrm{~Gy}$ in 3 fractions and 3 days ( 9 patients), 2 series of 18Gy spaced 21 days apart (54 patients), 20Gy in 5 fractions and 5 days ( 3 patients), or 30Gy in 10 fractions (5 patients). In this palliative treatment class, chemotherapy was administered to 8 patients, 7 in concomitant radiochemistry with Temozolomide and 1 patient according to the carboplatin/etoposide regimen.

\section{Prognosis factors}

In univariable analysis, survival was significantly associated with age, histology, grade, surgery and oncological treatment. In multivariable analysis, the same parameters were predictors of outcome, except surgery (Table $3)$.

Table 3: Prognosis factors from the univariable and multivariable analyses

\begin{tabular}{c|ccc|ccc} 
& \multicolumn{3}{|c|}{ Univariable } & \multicolumn{3}{c}{ Multivariable } \\
\hline Parameters & HR & p-value & $95 \%$ CI & HR & p-value & $95 \%$ CI \\
\hline Age & 1.058 & $\mathbf{1 . 7 9 e - 1 4}$ & $1.04-1.07$ & 1.032 & $\mathbf{0 . 0 0 0 2 6}$ & $1.01-1.05$ \\
Sex & 1.129 & 0.495 & $0.79-1.60$ & 1.159 & 0.414 & $0.81-1.65$ \\
Location & 0.619 & 0.061 & $0.37-1.02$ & 0.921 & 0.729 & $0.58-1.47$ \\
Histology & 0.809 & $\mathbf{0 . 0 0 1 4}$ & $0.71-0.92$ & 0.759 & $\mathbf{0 . 0 0 3 7}$ & $0.63-0.91$ \\
Grade & 3.055 & $\mathbf{1 . 9 e - 1 5}$ & $2.32-4.02$ & 2.464 & $\mathbf{5 . 3 e - 0 9}$ & $1.82-3.34$ \\
Surgery & 0.771 & $\mathbf{0 . 0 2 9}$ & $0.61-0.97$ & 0.980 & 0.872 & $0.77-1.25$ \\
Oncological & 3.115 & $<\mathbf{2 e - 1 6}$ & $2.55-3.81$ & 3.365 & $<\mathbf{2 e - 1 6}$ & $2.66-4.25$ \\
treatment & & & & & &
\end{tabular}

HR: Hazard Ratio

\section{Survival curves}

Figure 1 represents Kaplan-Meier survival curves according to age groups, location of tumors, histological subtype and grade of malignancy. It showed that survival probability decreases with age and grade of malignancy, that improved survival was observed in infra-tentorial tumors and that the worst prognosis was seen in GBM patients. 

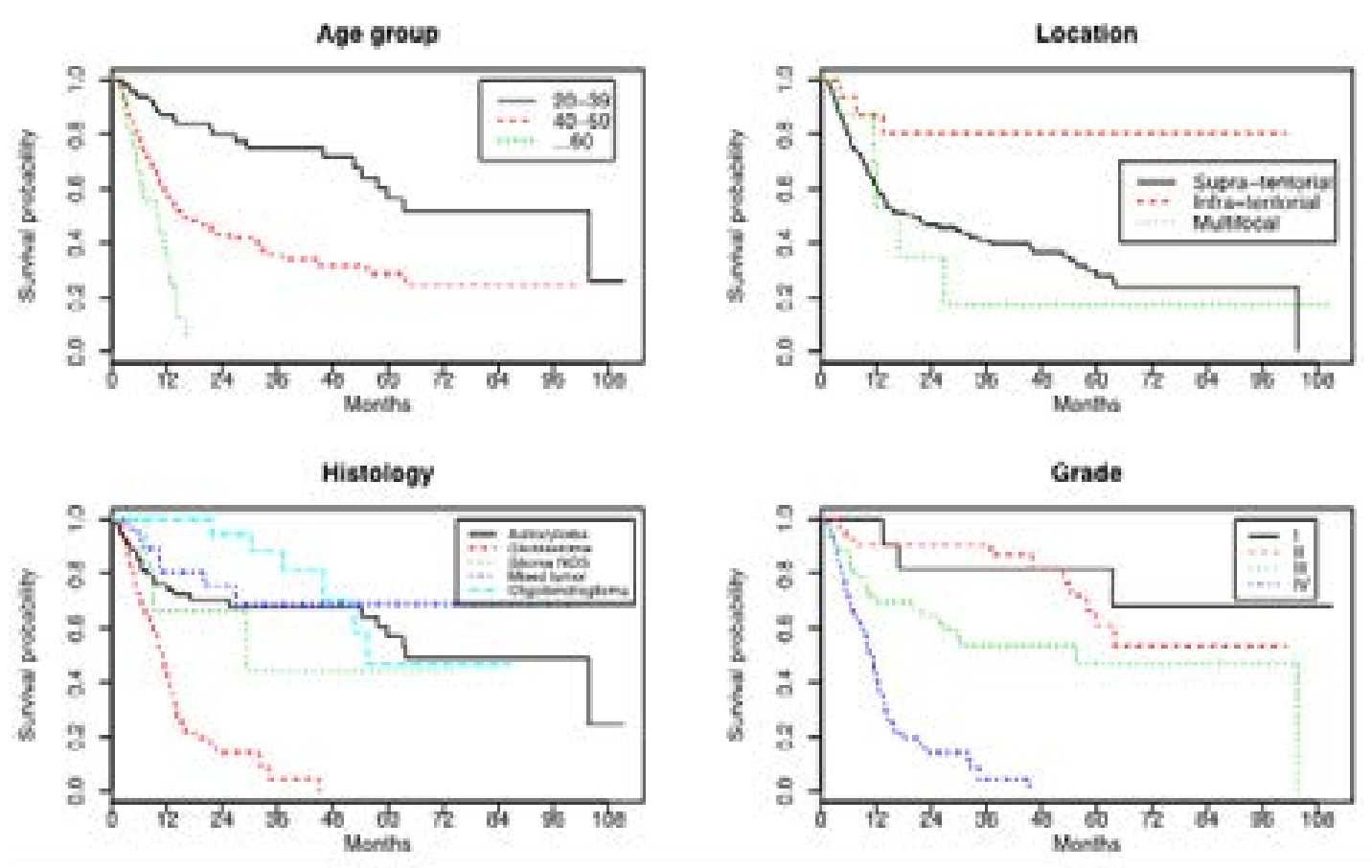

Figure 1 : Kaplan-Meier survival curves according to: Age groups, Tumor location, Histology and Grade of malignancy

\section{Testing Proportional Hazard Assumption}

The test of proportionality assumption was done using the Schoenfeld residuals. Table 4 shows that age, sex, location, and type of surgery meet assumptions Proportional Hazard with p-value $>0.05$. Meanwhile, the variables histological subtype, grade of malignancy and oncological treatment did not fulfill the assumption with $\mathrm{p}$-values of $0.04,0.001$ and 0.0003 respectively. Based on these results, an extended cox model was required.

Table 4: Correlation, $\chi^{2}$ and $p$-values of proportionality assumption test

\begin{tabular}{c|ccc} 
Parameters & Rho & $\chi^{2}$ & $\mathrm{p}$ \\
\hline Age & -0.029 & $1.42 \mathrm{e}-01$ & 0.71 \\
Sex & -0.0015 & $3.09 \mathrm{e}-04$ & 0.98 \\
Location & 0.034 & $2.06 \mathrm{e}-01$ & 0.65 \\
Histology & $\mathbf{0 . 1 8 4}$ & $\mathbf{4 . 2 1 e}+\mathbf{0 0}$ & $\mathbf{0 . 0 4}$ \\
Grade & $\mathbf{0 . 1 9 2}$ & $\mathbf{6 . 5 1 e}+\mathbf{0 0}$ & $\mathbf{0 . 0 1 1}$ \\
Surgery & -0.027 & $1.04 \mathrm{e}-01$ & 0.75 \\
Oncological treatment & $\mathbf{- 0 . 3 2 4}$ & $\mathbf{1 . 3 0 e}+\mathbf{0 1}$ & $\mathbf{0 . 0 0 0 3}$
\end{tabular}

Cox Extended Proportional Hazard (PH) model To overcome the variables that do not meet the cox proportional hazard assumption, a new model "Cox
Extended" was fitted using the "Step functions" method. The prognosis factors from the new model are shown in table 5. 
Table 5: prognosis factors from the Extended Cox-PH

\begin{tabular}{c|cccc} 
Parameters & HR & SE & $\mathrm{p}$ & $95 \% \mathrm{CI}$ \\
\hline Age & $\mathbf{1 . 0 2 1}$ & $\mathbf{8 . 4 4 e - 0 3}$ & $\mathbf{0 . 0 1 3}$ & $\mathbf{1 . 0 0 4 - 1 . 0 3 8}$ \\
Sex & $\mathbf{1 . 4 8 0}$ & $\mathbf{1 . 9 1 e - 0 1}$ & $\mathbf{0 . 0 3 9}$ & $\mathbf{1 . 0 1 8 - 2 . 1 5 1}$ \\
Location & 0.923 & $2.28 \mathrm{e}-01$ & 0.726 & $0.591-1.444$ \\
Histology & $\mathbf{0 . 7 4 6}$ & $\mathbf{1 . 0 6 e - 0 1}$ & $\mathbf{0 . 0 0 6}$ & $\mathbf{0 . 6 0 7 - 0 . 9 1 8}$ \\
Grade & $\mathbf{1 . 5 1 1}$ & $\mathbf{1 . 5 4 e - 0 1}$ & $\mathbf{0 . 0 0 7}$ & $\mathbf{1 . 1 1 8 - 2 . 0 4 2}$ \\
Surgery & 0.82 & $1.39 \mathrm{e}-01$ & 0.153 & $0.625-1.077$ \\
Oncological treatment & & & & \\
Strata 1 & $\mathbf{3 . 8 0 5}$ & $\mathbf{1 . 6 2 e - 0 1}$ & $<\mathbf{2 e - 1 6}$ & $\mathbf{2 . 7 6 8 - 5 . 2 2 9}$ \\
Strata 2 & 1.357 & $3.80 \mathrm{e}-01$ & 0.422 & $0.644-2.858$ \\
Strata 3 & $\mathbf{5 . 1 4 5}$ & $\mathbf{8 . 3 4 e - 0 1}$ & $\mathbf{0 . 0 4 9}$ & $\mathbf{1 . 0 0 4 - 2 6 . 3 7}$ \\
Strata 4 & $1.85 \mathrm{e}-06$ & $2.49 \mathrm{e}+03$ & 0.996 & $0.0000-$ Inf \\
HR: Hazard Ratio/ SE : Standard Eror & & & &
\end{tabular}

From table 5, we can see that age, histological subtype and grade of malignancy remain associated with the survival of patients, while the oncological treatment was found to be time dependent, and sex, not associated in the first model, has shown a significant $\mathrm{p}$-value. The assumption of the extended-cox model was met with a global p-value of 0.5724 .

\section{Discussion}

Glial tumors encompass a large group of histological subtypes, mainly occurring in the frontal, temporal, parietal, and occipital lobes combined (60.8\%). Only a very small proportion of gliomas occurred outside the brain ${ }^{4}$. Distributions of these tumors vary substantially by age group and sex ${ }^{4,9}$. Survival of these pathologies generally decreases with older age at diagnosis; children and young adults mostly have better survival for most glioma types ${ }^{4}$.

In this retrospective study, descriptive epidemiologic data and survival analysis. The results revealed a young population with a relatively good survival rates.

The demographic data showed a male preponderance with a sex ratio male / female of 1.87 , which is consistent with what is known for glial tumors ${ }^{4,9-11}$. The relation between patients' sex and the other characteristics did not show any statistical significance.

Concerning age, the Algerian glioma population (mean $=48$, median $=49$ years) was found younger than the French (median $=58$ years) and the American (mean $=51.9$ ), close to the Tunisian (median $=48$ ) and older than the Chinese (median $=41)^{11-14}$. Moreover, the decrease of the number of observed cases in the old adult age group is also reported in the Tunisian, and the Asian populations and is in contrast with the Euro- pean, the American and the Canadian findings, where no decrease was observed ${ }^{4,6,11,15-17}$. This difference may be explained by variations in life expectancy at birth between developed and developing countries or by disparity in availability of health care ${ }^{18}$.

Regarding the distribution of tumor location and histological subtypes, and as expected from previous studies, GBM with $55 \%$ of cases was the most frequent histological subtype, and supra-tentorial location (91.9\%) the most represented one ${ }^{4,12,15,17,19}$. Multifocal tumors and Glioma NOS were the less represented types with the worst prognosis for the first and the worst after GBM for the second. Furthermore, correlations between age, location and grade of malignancy reported in this study are supported by the pattern: Low Grade Glioma (LGG) and infra-tentorial tumors are more frequent within the youngest patients while High Grade Glioma (HGG) and supra-tentorial lesions are the most frequent among the oldest ${ }^{19-23}$.

The majority of our patients received a large resection and a curative protocol of oncological treatment. The current strategy for treatment of gliomas consists of a maximal surgical resection, an external beam radiation therapy (the standard dose is 60 gray (Gy), delivered in 30 to 33 fractions of $1.8-2(\mathrm{~Gy})$ ) and chemotherapy (Temozolamide: an oral cytotoxic DNA-alkylating) ${ }^{24}$. The carboplatin/etoposide regimen was used when the Temozolamide was not available.

The survival pattern of our population joins the other populations in several points such as the slight better outcome for women compared to men ${ }^{6,25}$, the decrease survival with increasing age ${ }^{6,11}$, and increasing grade of malignancy ${ }^{14,19}$ and survival of GBM ${ }^{17,26-28}$. Nonetheless, the minor fluctuations observed when comparing the cohort to others become more accentuated when it 
comes to the OS (mean $=45.4$ months, median $=21.7$, survival rates at 1-, 2-, and 5-years of $62.8 \%, 48.5 \%$ and $32.9 \%$ respectively) found to be higher than the European $(1-$ and 5 - years survival $=56.9 \%$ and $19.6 \%$, respectively) and the Tunisian (mean $=27$ months, 1 -, 2 - and 5- years survival rates : $42 \%, 30 \%$ and $26 \%$ respectively) $6,11,14,17,19,26$. This may be explained in part by the 17 patients who lived more than 60 months (up to 120 months).

The improved survival rate of infratentorial tumors may be explained by its distribution, in fact this class was mainly composed of low grade glioma (16 of 18) in which astrocytoma was the major histological subtype (13 low grade astrocytoma). It was reported that the histological grade in gliomas of the posterior fossa is the main prognostic factor ${ }^{29}$. Moreover adults with cerebellar WHO Grade I and II astrocytomas have been described as having a much more favorable survival curve than those with similar supratentorial tumors ${ }^{30}$.

The results concerning the prognosis factors showed that the age and the pathological type were significantly associated with the survival rate, which is in agreement with what was reported in several studies ${ }^{14,31}$. The type of surgery, which showed statistical significance only in the univariable analysis of the first model, was not associated with the outcome of patients. Furthermore, the data has revealed that, surprisingly, patients who had a partial resection lived longer than those who underwent a large resection. These findings contradicts with the largely documented superior efficacy of gross total resection over sub-total resection on survival, functional outcome, tumor progression and malignant transformation in patients with both low and high grade gliomas ${ }^{31-35}$. This difference cannot be explained by an eventual link between the extent of resection and age or histological subtype since there is none.

The oncological treatment was also found to be correlated with survival in the cohort; however, it was found to be time dependent in the new model. The extended cox model has also highlighted an unexpected factor which is the sex of the patients. It was not associated before and the survival of glioma was not linked to the sex in the literature. Since the aim is not to evaluate any model or technique, we can only conclude that the oncological treatment with the age and pathological type are associated with the survival in our population.

In the present study, there were some limitations like the low follow up time, the lack of time of recurrence from records that could have allowed us to calculate
Progression Free Survival (PFS), and the performance status. Another limitation was the low number of studies on gliomas in Arab Maghreb and Africa with which one could make comparisons. The approach of "available case" (or listwise deletion) described by Kang ${ }^{36}$ was used for incomplete records, simply all cases with missing data were removed from the analyses.

Despite all these limitations, the report brings insights into glioma epidemiology in Algeria and constitutes a sample of the Maghreb and African populations. To our knowledge, this is one of the first studies of glioma epidemiology in Algeria. Identifying features of gliomas in such populations may complete our comprehension of this cancer and may constitute a starting point for larger investigations in the country. Moreover, these results can inform clinicians about the characteristics of our population and assist them in making decisions.

\section{Conclusion}

In summary, 333 cases of patients with glioma brain tumors from Eastern Algeria were studied and the cohort was relatively young with a higher survival than others. Patients who had a partial resection had a better survival than those who underwent a large resection. Age, histological subtype, grade of malignancy and oncological treatment strategy were the most important predictive factors of outcome.

More investigations are required to explain the longer survival among patients with subtotal resection as well as further molecular analyses, in order to better characterize and complete the glioma profile for the Algerian population and, consequently, improve treatment strategies and outcomes of glioma patients.

\section{Conflict of interest}

The authors declare that they have no conflict of interest.

This research did not receive any specific grant from funding agencies in the public, commercial, or not-forprofit sectors.

\section{References}

1. Ostrom, Q. T., Gittleman, H., Stetson, L., Virk, S. M. \&Barnholtz-Sloan, J. S. Epidemiology of Gliomas. in Current Understanding and Treatment of Gliomas (eds. Raizer, J. \&Parsa, A.) 1-14 (Springer International Publishing, 2015). doi:10.1007/978-3-319-12048-5_1.

2. KOMORI, T. The 2016 WHO Classification of Tumours of the Central Nervous System: The Major 
Points of Revision. Neurol Med Chir (Tokyo)57, 301311 (2017).

3. Bray, F. et al. Global cancer statistics 2018: GLOBOCAN estimates of incidence and mortality worldwide for 36 cancers in 185 countries. CA: A Cancer Journal for Clinicians. 68, 394-424 (2018).

4. Ostrom, Q. T. et al. CBTRUS Statistical Report: Primary brain and other central nervous system tumors diagnosed in the United States in 2010-2014. Neuro-oncology. 19, v1-v88 (2017).

5. Louis, D. N. et al. The 2007 WHO Classification of Tumours of the Central Nervous System. Acta Neuropathol. 114, 97-109 (2007).

6. Crocetti, E. et al. Epidemiology of glial and non-glial brain tumours in Europe. European Journal of Cancer. 48, 1532-1542 (2012).

7. Ferlay, J. et al. Cancer incidence and mortality worldwide: sources, methods and major patterns in GLOBOCAN 2012. Int. J. Cancer. 136, E359-386 (2015).

8. Hamdi Cherif, M. et al. Time trends of cancer incidence in Setif, Algeria, 1986-2010: an observational study. BMC Cancer. 14, 637 (2014).

9. Bondy, M. L. et al. Brain tumor epidemiology: consensus from the Brain Tumor Epidemiology Consortium. Cancer. 113, 1953-1968 (2008).

10. Sehmer, E. A. J. et al. Incidence of glioma in a northwestern region of England, 2006-2010. Neuro Oncol. 16, 971-974 (2014).

11. Trabelsi, S. et al. Glioma epidemiology in the central Tunisian population: 1993-2012. Asian Pac. J. Cancer Prev. 15, 8753-8757 (2014).

12. Darlix, A. et al. Epidemiology for primary brain tumors: a nationwide population-based study. J. Neurooncol.131, 525-546 (2017).

13. Deb, S. et al. The effect of socioeconomic status on gross total resection, radiation therapy and overall survival in patients with gliomas. J. Neurooncol. 132, 447 453 (2017).

14. Yang, P. et al. Management and survival rates in patients with glioma in China (2004-2010): a retrospective study from a single-institution. J. Neurooncol.113, 259266 (2013).

15. Dho, Y.-S. et al. An Updated Nationwide Epidemiology of Primary Brain Tumors in Republic of Korea, 2013. Brain Tumor Res Treat. 5, 16-23 (2017).

16. Jazayeri, S. B., Rahimi-Movaghar, V., Shokraneh, F., Saadat, S. \&Ramezani, R. Epidemiology of primary CNS tumors in Iran: a systematic review. Asian Pac. J. Cancer Prev. 14, 3979-3985 (2013).
17. Yuan, Y., Ross, J., Shi, Q. \& Davis, F. G. Conditional survival after a diagnosis of malignant brain tumour in Canada: 2000-2008. Curr Oncol. 24, e341-e347 (2017).

18. WHO | World Health Statistics 2016: Monitoring health for the SDGs. WHO http://www.who.int/gho/ publications/world_health_statistics/2016/en/.

19. Rasmussen, B. K. et al.Epidemiology of glioma: clinical characteristics, symptoms, and predictors of glioma patients grade I-IV in the the Danish Neuro-Oncology Registry. J. Neurooncol. 135, 571-579 (2017).

20. Bhurgri, Y. et al. Trends and morphology of central nervous system malignancies in Karachi. Asian Pac. J. Cancer Prev. 12, 2013-2017 (2011).

21. Dobes, M. et al. A multicenter study of primary brain tumor incidence in Australia (2000-2008). Neuro-oncology. 13, 783-790 (2011).

22. McKean-Cowdin, R. et al. Trends in childhood brain tumor incidence, 1973-2009. J. Neurooncol.115, 153-160 (2013).

23. Johnson, K. J. et al. Childhood Brain Tumor Epidemiology: A Brain Tumor Epidemiology Consortium Review. Cancer Epidemiol Biomarkers Prev. 23, 2716-2736 (2014).

24. Bush, N. A. O., Chang, S. M. \& Berger, M. S. Current and future strategies for treatment of glioma. Neurosurg Rev. 40, 1-14 (2017).

25. Indirect costs associated with glioblastoma: Experience at one hospital. - PubMed - NCBI. https://www. ncbi.nlm.nih.gov/pubmed/27449154.

26. ALTWAIRGI, A. K. et al.Outcome of patients with glioblastoma in Saudi Arabia: Single center experience. Mol Clin Onco. 14, 756-762 (2016).

27. Brodbelt, A. et al. Glioblastoma in England: 20072011. Eur. J. Cancer. 51, 533-542 (2015).

28. Fuentes-Raspall, R. et al.Descriptive epidemiology of primary malignant and non-malignant central nervous tumors in Spain: Results from the Girona Cancer Registry (1994-2013). Cancer Epidemiology. 50, 1-8 (2017).

29. Strauss, I. et al. Gliomas of the posterior fossa in adults. J. Neurooncol.115, 401-409 (2013).

30. Bagley, J. H., Babu, R., Friedman, A. H. \& Adamson, C. Improved survival in the largest national cohort of adults with cerebellar versus supratentorial low-grade astrocytomas. Neurosurg Focus. 34, E7 (2013).

31. Wrensch, M. et al. Diagnostic, treatment, and demographic factors influencing survival in a population-based study of adult glioma patients in the San Francisco Bay Area. Neuro-oncology. 8, 12-26 (2006). 
32. Sanai, N. \& Berger, M. S. Glioma extent of resection and its impact on patient outcome. Neurosurgery. 62, 753-764; discussion 264-266 (2008).

33. Amirian, E. S., Armstrong, T. S., Aldape, K. D., Gilbert, M. R. \&Scheurer, M. E. Predictors of survival among pediatric and adult ependymoma cases: a study using Surveillance, Epidemiology, and End Results data from 1973 to 2007. Neuroepidemiology. 39, 116-124 (2012).
34. Tang, S., Liao, J. \& Long, Y. Comparative assessment of the efficacy of gross total versus subtotal total resection in patients with glioma: A meta-analysis. Int J Surg. 63, 90-97 (2019).

35. Ding, $X$. et al. The prognostic value of maximal surgical resection is attenuated in oligodendroglioma subgroups of adult diffuse glioma: a multicenter retrospective study. J. Neurooncol.40, 591-603 (2018).

36. Kang, $\mathrm{H}$. The prevention and handling of the missing data. Korean J Anesthesiol. 64, 402-406 (2013). 